\title{
Power System Reconfiguration Using Graph Trace Analysis and Multi-agent System
}

\author{
Anli Shang \\ a College of Electrical Engineering \\ Naval University of Engineering \\ Wuhan, China \\ E-mail: sxsal001@sina.com \\ b State Key Laboratory of Electrical Insulation and \\ Power Equipment \\ Xi'an Jiaotong University \\ Xi' an, China
}

\author{
Shengmao Yan \\ College of Electrical Engineering \\ Naval University of Engineering \\ Wuhan, China \\ E-mail: 5616729@qq.com
}

\begin{abstract}
This paper presents a multi-agent system that performs shipboard power system reconfiguration. If shipboard electric power supply interruption is caused by a fault, it is important to restore the power system as soon as possible to a target network configuration after the fault. The system views the problem as the interaction of independent agents. The approach is implemented using MAS (Multi Agent System) and graph trace analysis (GTA) method. Simulation results have demonstrated that the method is able to reach the target configurations.
\end{abstract}

Keywords-Shipboard Power System; Multi-agent System; Graph Trace Analysis; Reconfiguration; Iterator

\section{INTRODUCTION}

The shipboard power system (SPS) provides energy to electrical loads on ship for navigation, communication, operation and so on. The reliability of power supply is important for SPS. When a fault occurs on a ship power system, the protection systems will isolate the fault from the remaining power grid. Then the system should restore the electric power to a target configuration after the outage. Various methods have been applied to the reconfiguration process of power systems [1-4]. However, most of the proposed reconfiguration methodologies are centralized. In a Shipboard Power System's centralized reconfiguration approach, a single point of failure may happen if the system lacks redundancy.

MAS are ideal for control of energy resources to achieve higher reliability, and more efficient (optimum) power generation and consumption. Because MAS processes data locally and only transfer results to an integration center, computation time is largely reduced, and the network bandwidth is very much reduced compared to that of a central control [2]. GTA is used for the design and planning of electric power systems and is also used as a model-based analysis engine for real-time control and operations, including control of distributed generation [5].

In this paper, a novel service restoration approach for complex SPS is proposed based on the graph trace analysis and multi-agent system.

The shipboard system is showed in Section II, Section III introduces multi-agent system and GTA technique, and Section IV discusses the framework for multi-agent restoration. In Section V, three test cases are illustrated. Section VI draws the conclusions.

\section{SHIPBOARD POWER SYSTEM RESTORATION PROBLEM}

\section{A. System Objective and Constraints for Restoration}

The objective of a power system restoration is to maximize the capacity of the served loads

$$
\max \sum_{\mathrm{k} \in R} L_{k} y_{k}
$$

where $L_{k}$ is the load at bus $\mathrm{k}, y_{k}$ is the decision variable of expressing its status ( $y_{k}=1$ : restored; $y_{k}=0$ : not restored), and $R$ denotes the set of deenergized loads. And there are several typical constrains for this model:

a. There is a limit for the available capacity for system restoration;

b. The supply and demand power must be balanced;

c. This constraint used is mandatory in the real power system operations.

\section{B. Shipboard Reference System}

A schematic of a typical shipboard power system is shown in Fig. 1. The system consists of two AC generators, two AC propulsion loads, AC to DC converters, and DC distribution system. The loads are divided into three zones. Zonal distribution reduces the amount of cabling and watertight penetrations required, and simplifies damage control and maintenance. Each zone generally contains one or more load centers that supply power to electric loads in that zone. The overall DC distribution system is divided into two DC buses viz. port bus and starboard bus. These buses can be supplied from any of the main generator units through a Power Supply (PS). Breakers are used to isolate zones from each other. Each zone may contain an alternate power source and the load centers are capable of supplying normal or alternate power to the loads. Each zone has two converter units, each connected to one bus. These units supply power to zonal loads [6]. 


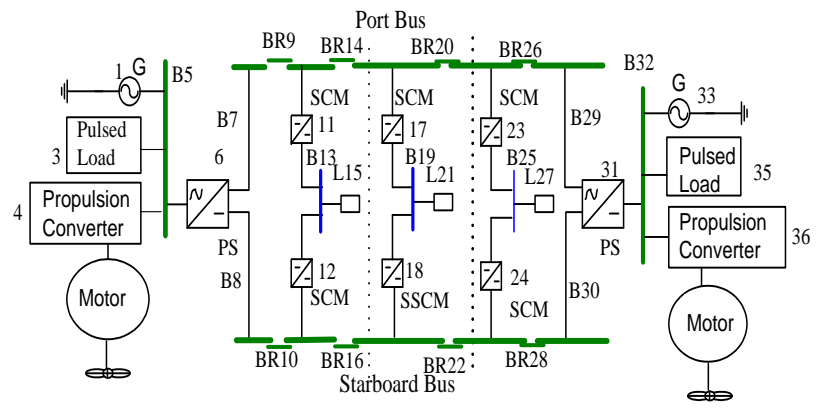

Figure 1. Shipboard power system where G:Generator,PS: Power Supply, SCM: Service Converter Module, B:Bus ,L:Load, BR:Breaker.

\section{Multi-Agent Based GTA Technique}

\section{A. Multi-Agent System (MAS)}

An agent is an abstraction object (software or hardware) capable of autonomous action in some environment. In this environment, an agent is an information processor that performs autonomous actions based on environment information. MAS are a computational system, in which several agents cooperate to achieve some tasks. The performance of multi-agent systems can be decided by the interactions among various agents. Agents cooperate so that they can achieve more than they would if they act individually. Multi-agent systems are more than a systems integration method; they also provide a modeling approach. By offering a way of viewing the world, an agent system can intuitively represent a real-world situation of interacting entities, and give a way of testing how complex behaviors may emerge.

\section{B. GTA Technique}

Graph Trace Analysis (GTA) is a method of analyzing systems predicated on graphs [5]. There is a one-to-one correspondence between each edge in the graph and each item in the physical system. GTA can be used to model systems that contain millions of components, where entities in a system are modeled as objects. The two main ideas GTA borrows from Generic Programming are the use of a container to hold collections of objects and iterators to traverse through and operate on objects stored in a container. Each component object is assigned a standardized set of iterators that defines its relationships with other components stored in the container. As components are added, modified, deleted, or change state, their iterators are automatically updated. Five iterators are defined: forward (f), backward (b), feeder-path (fp), brother (br) and adjacent (adj) iterators. Automated traces through the system implemented with iterators are used to generate "trace" sets. Researchers apply five types of graph traces to construct the component restoration paths. These traces yield five basic sets as follow:

FTp $=$ Sequence of components in forward trace from component $\mathrm{p}$

$\mathrm{BTp}=$ Sequence of components in backward trace from component $\mathrm{p}$

FPTp $=$ Sequence of components in feeder path trace from component $\mathrm{p}$

BRTp $=$ Sequence of components in brother trace from component $\mathrm{p}$
ADJp $=$ Sequence of components in adjacent trace from component $p$

In this paper each component's object is built as an agent, which encapsulates a particular task or set of functionality, in a similar way to modular or objectoriented programming. The main benefit of the object approach is data-encapsulation: the particular data structures used to hold attributes of an object are hidden from external objects, but are indirectly accessible through method calls and standard interfaces[7]. Agents exhibit four key properties: autonomy (they operate without human intervention), sociality (they interact with other agents), reactivity (they perceive and react to their environment) and proactivity (they exhibit goal-oriented behavior by taking initiatives) [8]. The sociality connotes more than the simple passing of data between different agents, something many traditional systems do. It connotes the ability to negotiate and interact in a cooperative manner. That ability is normally underpinned by an agent communication language (ACL), which allows agents to converse rather than simply pass data.

\section{Multi-Agent Restoration Framework}

The power system (PS) is modeled as a graph, as shown in Fig. 2. The transformation of the block diagram is shown in Fig. 1. The graph model is achieved by representing components by edges in a graph. Each edge represents the component of breaker, bus, generator, converter or load respectively, also associate with an agent in software model.

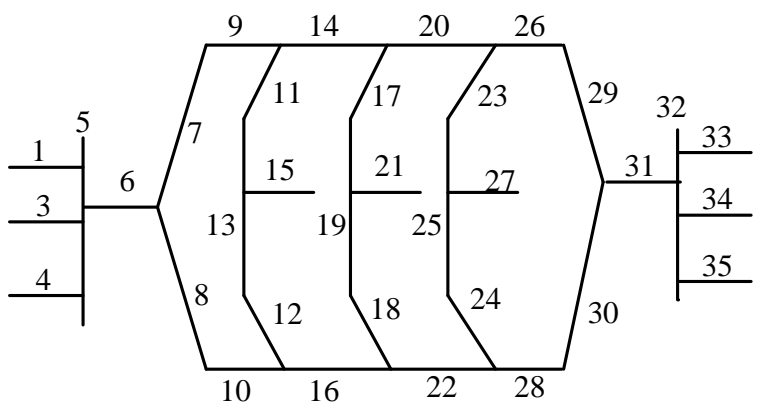

Figure 2. Integrated Power System GTA model

A Multi-Agent System (MAS) is proposed for the reasoning of the power system reconfiguration. In the MAS, each agent is associated with one electric component in the power system. According to the electric component to which an agent is associated, these agents in the MAS can be classified into five categories: generator

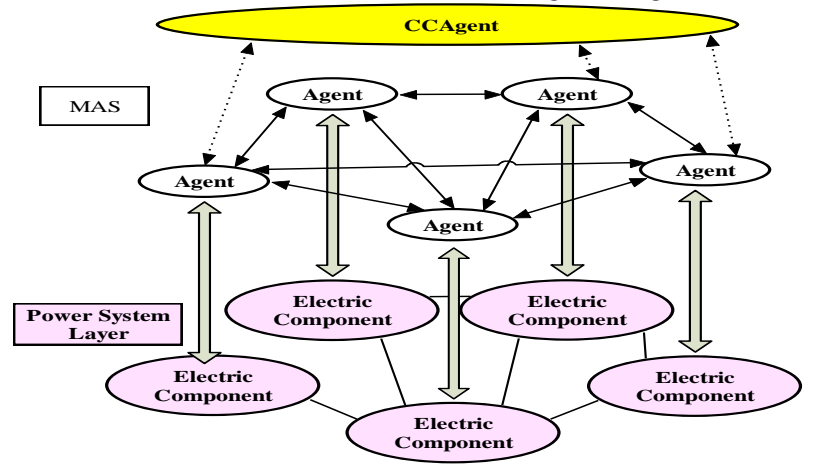

Figure 3. Agents and communication paths structure for power system 
Agent (GA), load agent(LA), breaker agent(BRA), converter module agent(CA) and bus agent(BA), which are in different levels. The whole system is divided as two subsystems, the communication sub system and operation subsystem. Fig. 3 illustrates the current and communication paths in system. The dotted line in Fig. 3 shows the communication paths between load agent and Command \& Control Agent (CCAgent). These agents are designed to report component status and require for restoration. The function of CCAgent is to maintain the negotiation process of the whole system. CCAgent receives the restoration request from unserved LA, builds an unserved LA list, and instigates the reconfiguration process by selecting corresponding agent.

Using GTA, the reconfiguration problem can be defined as follows. A component $\mathrm{C}$ is, for the purposes of restoration, a 16-tuple which defines certain characteristics of the component important to reconfiguration.

$\mathrm{C}=\{\mathrm{p}, \mathrm{type}, \mathrm{c}, \mathrm{f}, \mathrm{FT}, \mathrm{BT}, \mathrm{FPT}, \mathrm{BRT}, \mathrm{ADJ}, \mathrm{VLmin}, \mathrm{VLmax}$, $\mathrm{V}$, Pri,Status,Ap,Op

Where $\mathrm{p}=$ unique component identifier

type = Load, Circuit Breaker, Bus, Generator, Converter

$\mathrm{c}=$ capacity, or rating of a component

$\mathrm{f}=$ flow, where $\mathrm{f} \leqslant \mathrm{c}$

$\mathrm{ft}, \mathrm{fpt}, \mathrm{bt}, \mathrm{brt}, \mathrm{adjt}=\mathrm{components}$ related to $\mathrm{C}$ via forward, feeder path, backward, brother, and adjacent trace,respectively, where a value of 0 implies the component does not exist

VLmin,VLmax $=$ the maximum and minimum allowable voltage

Pri $=$ component priority

Status = status of component-ON, OFF, Failed

Op = Operable, whether the component can be turned on or off - YES, NO

The main objective in this restoration algorithm is to restore as many loads as possible by transferring deenergized loads via network reconfigurations to sources without violating operational constraints such as specified in the beginning of Section 2. The algorithm is implemented with topological iterators and graph trace sets. According to physical system topology, loads restoration path are already constructed and stored in corresponding agent.

A graph trace analysis (GTA) notation is introduced. The GTA notation builds on the Object Constraint Language (OCL) [9].

When there is a fault, after the fault isolation, the following procedures are proposed for ship system restoration.

Step 1 All the load agents that lost power report to the Command \& Control Agent (CCAgent).

Step 2 CCAgent creates the set of unserved load.

Step 3 CCAgent send a "start" message to one and only one agent to begin restoration based on pre-set priority list. When the number of agents in unserved load list is zero, go to step 11 .

Step 4 The selected agent (initiator) sends out a Call For Proposal (CFP) message, which specifies the task to be performed and a deadline for receiving replies, to $\mathrm{m}$ proposer agents that are included in the initiator's restoration path.
Step 5 If the responder agents are not able to perform the action e.g. their status are failed, or their excess power which is the difference between its maximum allowable power flow and its current power flow are no more than zero, or they are load components, the responder agents will send a Refusal message to the initiator. If so, the path including this component is not valid. If it has not reached its limit, it will evaluate the CFP message received. If the requested power value exceed its excess capacity it will propose only what is possible, otherwise it will propose the full amount requested by the initiator.

Step 6 When timeout occurs or all replies has been received, it is checked by the initiator agent that if any proposals has been received. If this is the case the next step is to evaluate them, otherwise the behavior terminates, and the attempt to restore power has been unsuccessful. Go to step 11.

Step 7 If one path or several paths can deliver the full amount which the initiator requires, the initiator will accept these and send Accept Proposal message to the responder agents one by one in the opposite trace fashion, such that the first closed switch is the one closest to the component reference source.

Step 8 The initiator wait for Inform messages from these responder agents that it has send Accept Proposal messages. If so, restoring power has been successful. Initiator is restored, system constraints are checked for the components that have been affected by the restoration, go to step 9. Otherwise go to step 11.

Step 9 The energized load agent sends a "restored" message to CCAgent.

Step 10 The CCAgent deletes the "restored" load agent from unserved load agent list. Then go to step 3.

Step 11 Terminate the restoration, and the attempt to restore power has been unsuccessful. Go to step 5

\section{TESTCASES}

A number of simulations were performed for the proposed strategy. This section will show two typical test cases for single path for reconfiguration and several paths for reconfiguration, respectively. Researchers assume that the priority list for 15, 21, 27.

\section{A. Case I: Full restoration for fault}

Researchers assume faults on component 11 and 17; the load 15 and 21 shown in Fig. 4 has lost power. Real number near the load shows the power, the numbers in the parentheses adjacent to the bus represent the amount of power flow (left) and the available power capacity (right).The unit is $\mathrm{kW}$. Where $\bigcirc$ represents that the component Status is Failed, and node represents that the component Status is OFF.

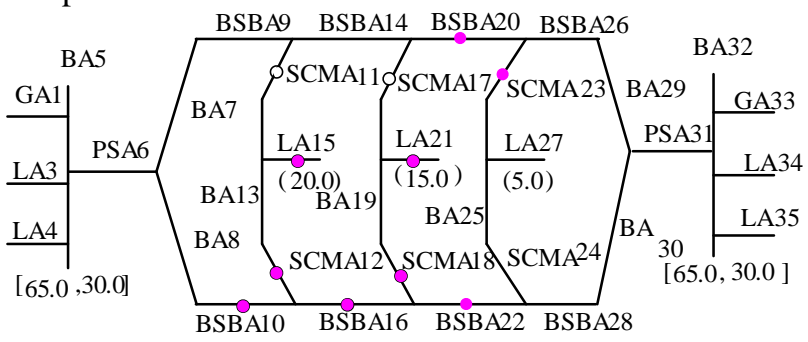

Figure 4. Network after fault for case I 
The approach first creates unserved load set $\{15,21\}$. Based on preset priority list, load 15 is selected to be first resorted. Because the distribution system is symmetrical, the backward component trace and the feeder path component trace are uniform. These traces beginning with component 15 yield four basic paths as follow:

$$
\mathrm{FPT} 115=\mathrm{LA} 15 \rightarrow \mathrm{BA} 13 \rightarrow \mathrm{SCMA} 11 \rightarrow \mathrm{BRA} 9 \rightarrow \mathrm{BA} 7 \rightarrow
$$

\section{$\mathrm{PSA} 6 \rightarrow \mathrm{BA} 5 \rightarrow \mathrm{GA} 1$}

$\mathrm{FPT} 215=\mathrm{LA} 15 \rightarrow \mathrm{BA} 13 \rightarrow \mathrm{SCMA} 12 \rightarrow \mathrm{BRA} 10 \rightarrow$

$\mathrm{BA} 8 \rightarrow \mathrm{PSA} 6 \rightarrow \mathrm{BA} 5 \rightarrow \mathrm{GA} 1$

$$
\text { ADJ115 }=\quad \text { LA15 } \rightarrow \text { BA13 } \rightarrow \text { SCMA11 } \rightarrow \text { BRA14 } \rightarrow
$$

$\mathrm{BRA} 20 \rightarrow \mathrm{BRA} 26 \rightarrow \mathrm{BA} 29 \rightarrow \mathrm{PSA} 31 \rightarrow \mathrm{BA} 32 \rightarrow \mathrm{GA} 33$

$\mathrm{ADJ} 215=\mathrm{LA} 15 \rightarrow \mathrm{BA} 13 \rightarrow \mathrm{SCMA} 12 \rightarrow \mathrm{BRA} 16 \rightarrow \mathrm{BRA} 2$

$2 \rightarrow$ BRA28 $\rightarrow$ BA30 $\rightarrow$ PSA $31 \rightarrow$ BA $32 \rightarrow$ GA33

LA15 then sends CFP message to Agent BA13,SCMA11,SCMA12,BRA9,BA7, PSA6,BA5,GA1, BRA10,BA8,BRA14,BRA20,BRA26,BA29,PSA31,BA32 ,GA33,BRA16,BRA22,BRA28,BA30.Since the fault is still there, SCMA11 will send a refuse message to LA15. FPT115 and ADJ115 are invalid restoration pathes. Because available capacity of FPT215 and ADJ215 (30.0)is greater than the request capacity (20.0), both pathes can be considered as load 15 restoration path, but the number of FPT215 switches operated is less than the number of ADJ215 switches operated, researchers choose FPT215 as load 15 restoration path. Switching of switches are ordered in the opposite fashion, such that the first switching of element in paths is the one closest to c's reference source. By addressing them in this order, service is gradually restored to the system rather than all at once. System constraints are checked and no problems are found. Once load 15 obtains sufficient power, it will send a Inform message to CCAgent. CCAgent then deletes LA 15 from unserved set. Load 21 can also be restored similarly. The new network is shown in Fig. 5.

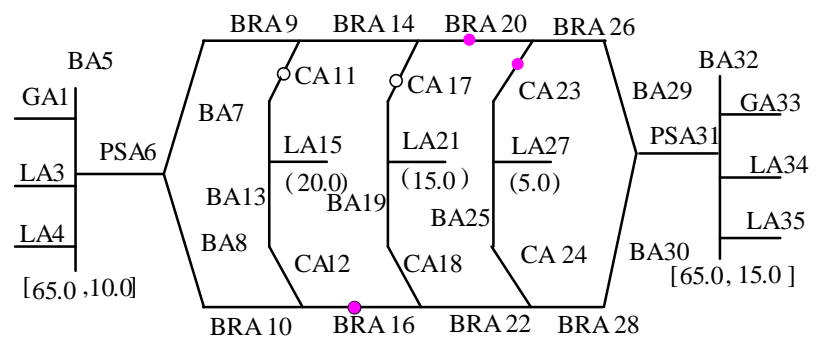

Figure 5. Network after reconfiguration for case I

\section{B. Case II:Multiple paths to complete a recovery task}

This case will show there is no one path that can independently restore the load power supply. With the same situation as Case I, there occur faults on component 11 and 17, the load 15 and 21 shown in Fig. 6 have lost power.

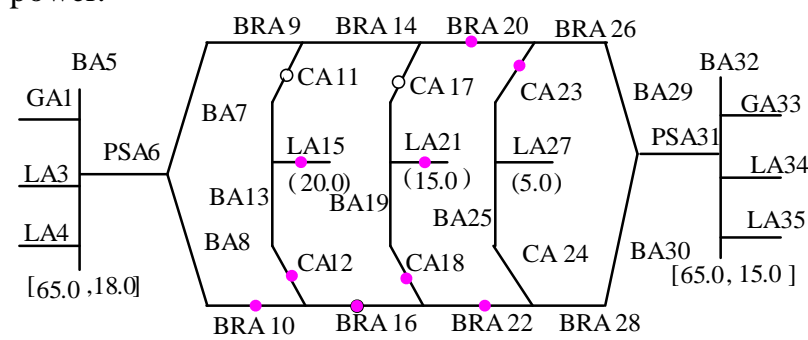

Figure 6. Post fault network for case II

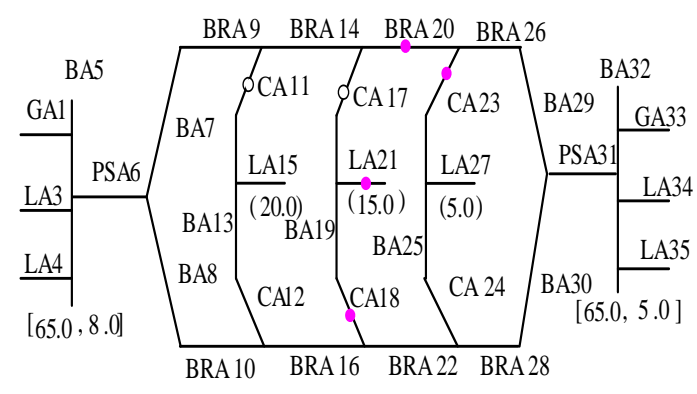

Figure 7. Network after reconfiguration for case II

\section{SUMMARY}

The concepts discussed in this paper represent a combination of concepts taken from Shipboard engineering, power utility engineering, and software engineering object-oriented analysis and design.

The paper provides a multi-agent-based approach for ship system electric power reconfiguration. The algorithm is designed to use a GTA model that is an unsimplified representation of the systems being analyzed, and is written using a new notation developed and described in this paper specifically for GTA. This notation can be used to write algorithms in GTA that are independent of the systems being analyzed. It is also easy to implement algorithms written this notation by using tools such as those provided in the JADE platform.

\section{ACKNOWLEDGMENT}

The authors wish to express their appreciation to National Natural Science Foundation of China for the support under the grant number "51177168"and supported by State Key Laboratory of Electrical Insulation and Power Equipment (EIPE15210)"). Researchers also appreciate the support from the staff and students of the college of electrical engineering, naval university of engineering.

\section{REFERENCES}

[1] M. E. Baran and F. F. Wu, "Network Reconfiguration in Distribution Systems for Loss Reduction and Load Balancing," IEEE Transaction on Power Delivery, Vol. 4, no. 2, 1989 ,pp. 1401-1407

[2] K. Huang, "Shipboard Power System Reconfiguration Using Multi Agent System," PhD Theses, The Florida State University,College of Engineering, 2007.

[3] S. Civanlar, J. J. Grainger, H. Yin, and S. S. H. Lee, "Distribution Feeder Reconfiguration for Loss Reduction," IEEE Transactions on Power Delivery, Vol. 3, no. 3, Jul. 1998, pp. 1217-1223.

[4] K. Nara, A. Shiose, M. Kitagawoa, and T, Ishihara, "Implementation of Genetic Algorithm for Distribution Systems Loss Minimum Reconfiguration," IEEE Transactions on Power Systems, Vol. 7, no. 3, Aug. 1992, pp. 1044-1051

[5] D.Kleppinger, R.Broadwater and C.Scirbona, "Generic Reconfiguration for Restoration Power System Research,"2010, pp. 287-295

[6] L.R. Feinauer, K.J. Russell, and R. Broadwater: "Graph trace Analysis and Generic Algorithms forInterdependent Reconfigurable System Design and Control," Naval Engineers Journal, Vol. 120, Iss. 1, March . 2008, pp. 29-40. DOI: $10.1111 /$ j.1559-3584. 
[7] S. D.Pekarek, Tichenor J., Sudhoff S. D., Sauer J.D., Delisle D. E., and Zivi E. J., "Overview of a naval combat survivability program," Proceedings of the 13th International Ship Control Systems Symposium, Orlando, Florida, 2003.

[8] S.McArthur, et.al., "Multi-Agent Systems for Power Engineering Applications-Part I: Concepts, Approaches, and Technical Challenges," IEEE Transactions on Power Systems, Vol. 22, No. 4, November 2007
[9] S. Franklin and A. Graesser, "Is it an agent, or just a program?: A taxonomy for autonomous agents," Proceedings of the third international workshop on agents, theories, architectures, and languages, pp. 21-35, 1996.

[10] Jos B. Warmer, Anneke G. Kleppe, "The Object Constraint Language: Precise Modeling With Uml[," Addison-Wesley Professional, 1st edition, 1998,Oct. 13. 